\title{
In situ compression tests on micron-sized silicon pillars by Raman microscopy-Stress measurements and deformation analysis
}

\author{
K. Wasmer ${ }^{a}$ \\ EMPA, Swiss Federal Laboratories for Materials Testing and Research, CH-3602 Thun, Switzerland \\ T. Wermelinger \\ Laboratory for Nanometallurgy, Department of Materials, ETH Zurich, 8093 Zürich, Switzerland \\ A. Bidiville \\ EMPA, Swiss Federal Laboratories for Materials Testing and Research, CH-3602 Thun, Switzerland \\ R. Spolenak \\ Laboratory for Nanometallurgy, Department of Materials, ETH Zurich, 8093 Zürich, Switzerland \\ J. Michler \\ EMPA, Swiss Federal Laboratories for Materials Testing and Research, CH-3602 Thun, Switzerland
}

(Received 16 May 2008; accepted 8 August 2008)

\begin{abstract}
Mechanical properties of silicon are of high interest to the microelectromechanical systems community as it is the most frequently used structural material. Compression tests on $8 \mu \mathrm{m}$ diameter silicon pillars were performed under a micro-Raman setup. The uniaxial stress in the micropillars was derived from a load cell mounted on a microindenter and from the Raman peak shift. Stress measurements from the load cell and from the micro-Raman spectrum are in excellent agreement. The average compressive failure strength measured in the middle of the micropillars is $5.1 \mathrm{GPa}$. Transmission electron microscopy investigation of compressed micropillars showed cracks at the pillar surface or in the core. A correlation between crack formation and dislocation activity was observed. The authors strongly believe that the combination of nanoindentation and micro-Raman spectroscopy allowed detection of cracks prior to failure of the micropillar, which also allowed an estimation of the in-plane stress in the vicinity of the crack tip.
\end{abstract}

\section{INTRODUCTION}

Characterization of the material properties of silicon (Si) in the micrometer and submicrometer range has become of particular interest in the last couple of years. This is because this material is widely used in thin film devices and microelectromechanical systems (MEMS), where size effects have been observed and examined by many researchers. This is especially the case for $\mathrm{Si}$ in the submillimeter ${ }^{1-3}$ and micrometer ${ }^{4}$ ranges. In these studies, mechanical properties were obtained from either bending or tensile tests. Recently, microcompression tests on Si micropillars with diameters ranging from 1 to $50 \mu \mathrm{m}$ were used to characterize the material properties and to study the size effects. ${ }^{5}$ The available literature on micropillar testing focuses on brittle rupture of Si rather

\footnotetext{
a) Address all correspondence to this author. e-mail: kilian.wasmer@empa.ch DOI: $10.1557 / J M R .2008 .0363$
}

than the general stress-strain $(\sigma-\epsilon)$ behavior. However, micro-Raman spectroscopy ( $\mu R S)$ is often used to study and/or measure the local mechanical stresses in silicon wafers and silicon-integrated circuits. ${ }^{6-8}$ The stress resolution of Raman microscopy in the case of silicon is $\sim 25 \mathrm{MPa} .{ }^{9}$ Furthermore, it is applied to investigate the microstructure of silicon. Indentation or scratching operations lead to phase transformations. The type of phases can be determined with Raman microscopy because of their unequal Raman spectra. ${ }^{10-14} \mathrm{An}$ other microstructural aspect that influences the Raman spectra is the grain size. Iqbal and Veprek showed that below a certain threshold $(\sim 10 \mathrm{~nm})$ the grain size of polycrystalline silicon can be determined from the Raman spectra. ${ }^{15}$ This work presents for the first time the combination of a $\mu \mathrm{RS}$ with a homemade microindenter to characterize the stress state localized in silicon micropillars during testing and to investigate the main deformation mechanisms in situ. The combination of these two techniques allows correlation of the mechanical 


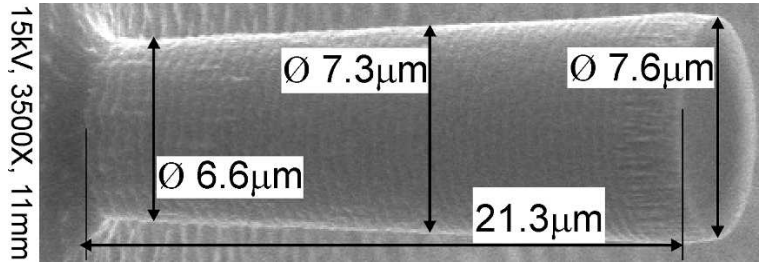

FIG. 1. SEM picture of a typical micropillar used for the microcompression test. The arrows indicate the approximate position of the diameter measurements.

properties with microstructural changes within the focus of the Raman laser during deformation.

\section{EXPERIMENTS AND MATERIALS}

Samples of $10 \times 10 \mathrm{~mm}^{2}$ were cut from a 4 in., $525 \mu \mathrm{m}$ thick (001)-oriented Si wafer. Cylindrical singlecrystalline silicon pillars were fabricated by standard photolithography techniques on the sample. A Heidelberg DWL200 (Heidelberg, Germany) direct laser writer was used to transfer the computer predefined design (with a square array of $10 \mu \mathrm{m}$ diameter circles) on the photoresist (Shipley Microposit S1800 series, Coventry, UK) coated wafer. The samples were obtained from silicon wafers just coated by a photoresist layer. After irradiation, and resist development, the wafers were anisotropically etched. To do this operation, a pulsed roomtemperature process (so-called Bosch process ${ }^{16}$ ) was applied. This is a cyclic process consisting of a few seconds of $\mathrm{Si}$ etching (by $\mathrm{SF}_{6}$ ) followed by a few seconds sidewall protection (by $\mathrm{C}_{4} \mathrm{~F}_{8}$ ), in the same plasma Alcatel 601 etcher (Annecy, France). When the etching process was completed, the residual photoresist was removed by a specific wet remover (Microposit Remover 1165, Shipley) and further cleaned by an oxygen plasma in a Branson IPC 2000 Plasma System. The method is fully described elsewhere. ${ }^{5}$

A typical pillar with its dimension is shown in Fig. 1. This figure shows that the diameter of the pillar varies slightly along its length due to the photolithography technique. Consequently, the geometry of all investigated pillars was measured by scanning electron microscopy (SEM) prior to deformation. The diameters were measured, at about half of the height of the micropillars, at the approximate location of the laser beam of the Raman microscope ( $\phi 7.3 \mu \mathrm{m}$ from Fig. 1). The length of all pillars was uniform. Their geometry and mean strength measurements (taken from Ref. 5) are summarized in Table I. The surfaces of the micropillars are not perfectly smooth as displayed in Fig. 1, which is inherent to the deep reactive ion etching process used. ${ }^{5}$

The Raman microscope used was a Confocal Raman Microscope CRM 200 (WITec GmbH, Ulm, Germany) with a helium cadmium laser with a wavelength of $442 \mathrm{~nm}$. All measurements were performed in backscattering mode without any filter. A $20 \times$ objective with a numerical aperture of 0.4 was used.

The microcompression experiments were performed using a homemade instrumented microindentation device developed to operate inside a SEM. This apparatus, fully described elsewhere, ${ }^{17}$ is based on a load cell (maximum load $P_{\max }$ of $500 \mathrm{mN}$ ) fixed on a piezoactuated positioning stage. In contrast to the description given in Ref. 17, a second stick slip stage was added to allow full Cartesian positioning of the specimen with respect to the indenter tip. The optical microscope of the Raman spectrometer was used to identify the micropillars. The load axis was inclined by $84^{\circ}$ to the microscope axis. The entire setup is shown in Fig. 2. The compression load was applied in the [001] direction, whereas the laser spotted the pillar at $90^{\circ}$ along the [010] direction. In such a setup, only the in-plane phonon gives rise to the Raman signal. Therefore, only the in-plane component of the stress tensor is measured. The corresponding shift of the Si Raman peak exhibits a stress sensitivity of $500 \mathrm{MPa} / \mathrm{cm}^{-1}$. 8,18

Compression tests were carried out using a diamond flat punch with a diameter of approximately $10 \mu \mathrm{m}$. The experiments were performed under constant displacement rate. The load on the sample, the displacement of the tip via the stack piezo, and the micro-Raman spectra were recorded simultaneously. The engineering stress, $\sigma$, was defined according to $\sigma=P / A$, where $P$ is the applied load and $A$ the initial cross section determined from SEM images. The cross-sectional area $A$ was calculated from the diameter in the middle of the height of the sample, as illustrated in Fig. 1, to correspond with the micro-Raman measurements.

To investigate the microstructure and the deformation mechanisms of the micropillars (phase transformations and/or crack initiation and propagation arising during the micromechanical tests), TEM lamellae of pillars after loading up to $200 \mathrm{mN}$ were prepared by focused ion beam (FIB; FEI Strata 235, Zürich, Switzerland) and observed with a Philips $\mathrm{CM} 30 \mathrm{LaB}_{6}$ TEM (Eindhoven,

TABLE I. Results of microcompression tests including the number of test, the geometry of the micropillars, and their mean strength measurement with its respective \pm 2 standard deviation (95\% confidence interval).

\begin{tabular}{|c|c|c|c|c|c|}
\hline $\begin{array}{c}\text { Location of diameter } \\
\text { measurement }\end{array}$ & $\begin{array}{l}\text { Number } \\
\text { of tests }\end{array}$ & $\begin{array}{c}\text { Sample } \\
\text { diameter }(\mu \mathrm{m})\end{array}$ & Length $(\mu \mathrm{m})$ & $\begin{array}{c}\text { Aspect ratio } \\
\text { (length/diameter) }\end{array}$ & $\begin{array}{c}\text { Mean compressive fracture } \\
\text { strength }(\mathrm{GPa})\end{array}$ \\
\hline Fracture & 27 & $6.6 \pm 0.06$ & 21.3 & 3.3 & $-7.0 \pm 0.3$ from Ref. 5 \\
\hline Middle & 20 & $7.3 \pm 0.06$ & 21.3 & 2.9 & $-5.1 \pm 0.4$ \\
\hline
\end{tabular}




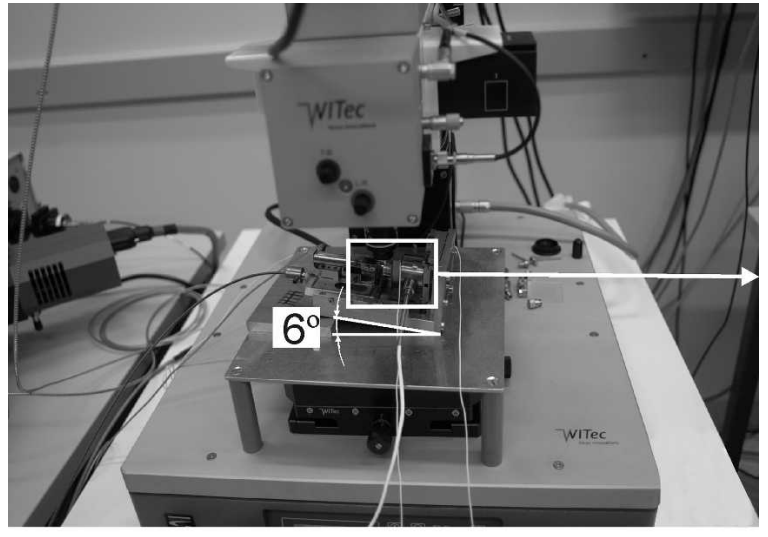

(a)

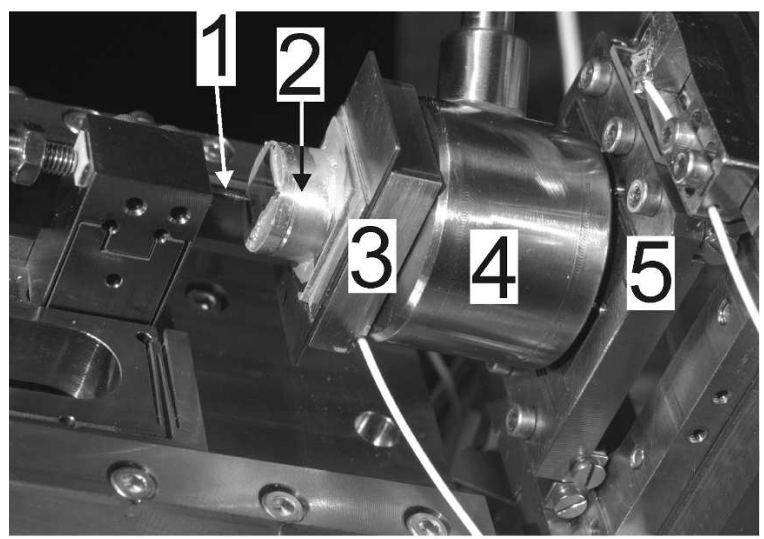

(b)

FIG. 2. Photographs showing (a) the experiments under the microRaman and (b) the setup of the compression experiment with (1) diamond tip, (2) specimen holder, (3) y-positioning stage, (4) load cell, and (5) $x$-positioning stage.

The Netherlands) using $300 \mathrm{kV}$ acceleration voltage. To distinguish the deformation process at the center of the pillar and on its side, one lamella was prepared in each location.

Forty microcompression tests of two types were conducted. First, 20 tests were loaded in purely elastic regime and unloaded, without holding time. The first 10 were loaded up to $140 \mathrm{mN}(\sigma=-3.3 \mathrm{GPa})$, whereas the maximum load for the next 10 experiments was $200 \mathrm{mN}$ $(\sigma=-4.6 \mathrm{GPa})$. Second, 20 tests were loaded until rupture. For each experiment, the loading and unloading rate was $1.0 \mathrm{mN} / \mathrm{s}$ and the force via the load cell and Raman measurements were recorded every $0.2 \mathrm{~s}$.

\section{RESULTS}

The results of the 20 tests until failure are summarized in Table I, where the mean compressive fracture strength in the middle of the pillar is $-5.1 \mathrm{GPa}$ with a standard deviation of \pm 0.4 . For the same tests set, the Weibull parameters were calculated; the Weibull modulus $m=$ 15.6, and the characteristic strength $\sigma_{0}=5.3 \mathrm{GPa}$.

Figure 3(a) displays a pillar that was compressed up to

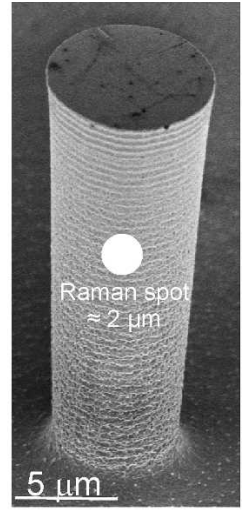

(a)

(b)
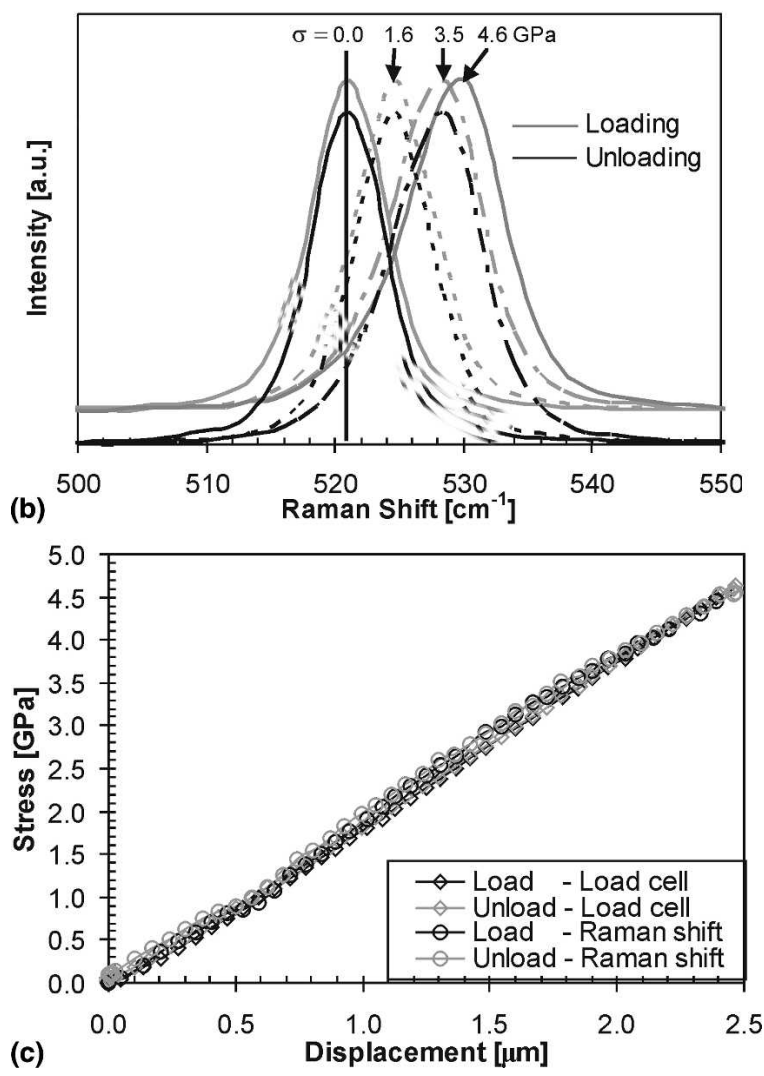

FIG. 3. Microindenter and micro-Raman results of an elastic micropillar compression test. (a) Micropillars subjected to a compressive stress of $-4.6 \mathrm{GPa}$ with the Raman spot. (b) Raman shift measured during loading-unloading with a velocity of $0.7 \mathrm{mN} / \mathrm{s}$ and a maximum load of $200 \mathrm{mN}$. The spectra intensity has been adjusted to facilitate the visual comprehension. (c) Comparison of the stress-displacement curve between both methods.

$-4.6 \mathrm{GPa}$ and unloaded. Inspection of this figure indicates that no crack or apparent damage is observed. The Raman spot was added to compare the spot with the pillar sizes. The corresponding plot of the Raman shift during the loading-unloading experiment is presented in Fig. 3(b). The Raman spectrum is given only between 500 and $550 \mathrm{~cm}^{-1}$ since no other peak is of further interest outside this range. The stresses, given in GPa, 
were derived from the load cell. The shift in the Raman spectra caused by the compressive stress is evident. No difference is observed between Raman spectra obtained, at identical stresses, during the loading and unloading sequence. This is also corroborated by the stressdisplacement curve in Fig. 3(c). This figure compares the compressive stress derived from (a) the load cell, based on the engineering stress, and (b) the Raman shift. The stresses derived from both methods are in very good agreement since the discrepancy between both methods throughout the test is less than $3 \%$. Finally, the lack of visible differences between the loading and unloading curves demonstrates that pillars compressed up to a load of $-4.6 \mathrm{GPa}$ behave purely elastically.

In three cases of the compression tests, a shoulder on the low-energy side of the silicon Raman peak was observed as illustrated in Fig. 4 by two examples. In Fig. 4(a), the shoulder appears in the spectrum at a compressive stress of $-1.87 \mathrm{GPa}$ and disappears during the measurement when $-2.47 \mathrm{GPa}$ were applied. The peak
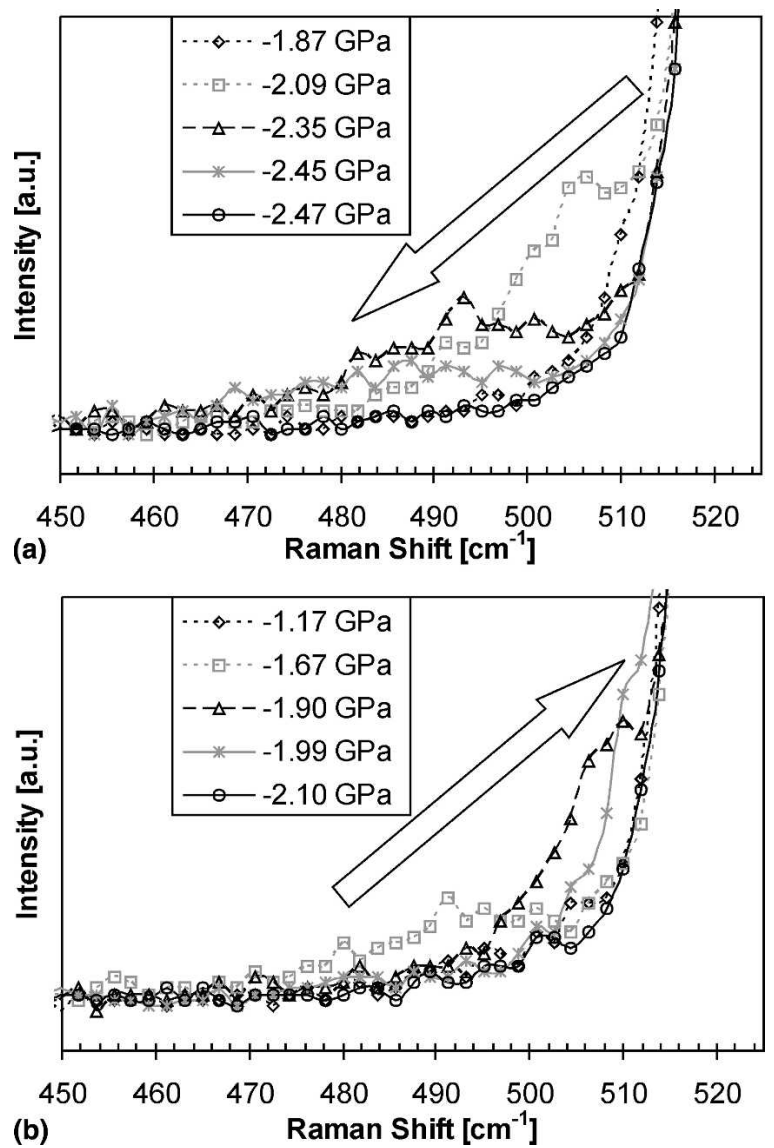

FIG. 4. Occurrence of a shoulder on the low-energy side of the Raman peak in two different tests. (a) Shoulder appears between -1.87 and $-2.47 \mathrm{GPa}$ stress. The peak position of the shoulder moves to lower wave numbers. (b) Shoulder appears between -1.17 and $-2.10 \mathrm{GPa}$ stress. The peak position of the shoulder moves to higher wave numbers. position of the shoulder moves to lower wave numbers, as indicated by the arrow, with increasing stress. The shape of the shoulder at a stress of $-2.45 \mathrm{GPa}$ and the shape of the curve at a stress of $-2.47 \mathrm{GPa}$ look different, although the applied stresses are almost equal. One has to keep in mind that $2 \mathrm{~s}$ have passed between the measurements of the two curves. In contrast, the shoulder in Fig. 4(b) starts to be visible at $-1.17 \mathrm{GPa}$ and vanishes at $-2.10 \mathrm{GPa}$. The arrow highlights that the peak position of the shoulder increases with increasing compressive stress. In both tests, the shoulder appears and disappears during the loading part of the test, although it moves in opposite directions in Figs. 4(a) and 4(b).

Figures 5 and 6 show two TEM lamellae machined with the [110] crystallographic direction normal to the foil. The lamella in Fig. 5(a) was cut in the middle of the pillar [see Fig. 5(b)] and shows several interesting features. The most obvious is a large crack initiated at approximately $600 \mathrm{~nm}$ under the surface and propagating to the center of the pillar to a maximal depth of $4.4 \mu \mathrm{m}$ in the pillar. This crack was already visible from the SEM image during the FIB preparation. It can be seen from the

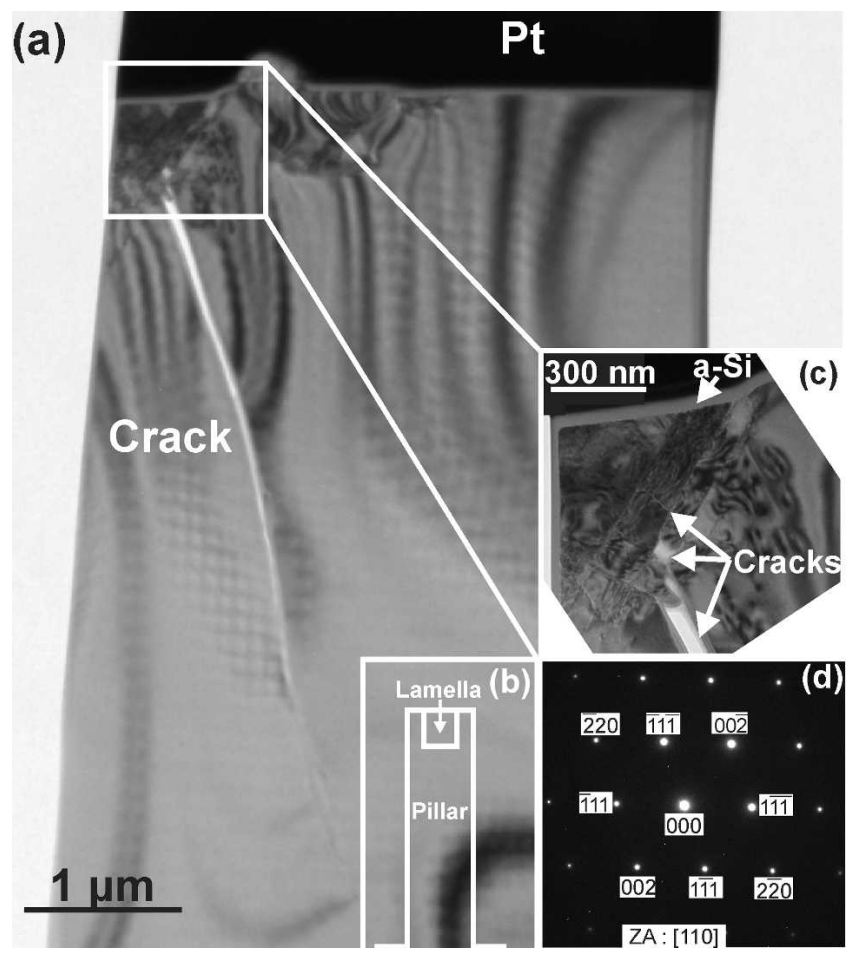

FIG. 5. TEM bright field ([110] projection) images of a FIB lamella made in the middle of a pillar subjected to a compressive stress of $-4.6 \mathrm{GPa}$. (a) Overview of the pillar with a crack starting approximately $600 \mathrm{~nm}$ under the surface and with a platinum layer at the surface. (b) Location of the FIB lamella in the middle of the micropillar. (c) The inset illustrates the location of the long cracks as well as small cracks along the few dislocations visible. (d) The diffraction pattern taken from the inset shows that no phase transformation occurred in this region during the experiments. 


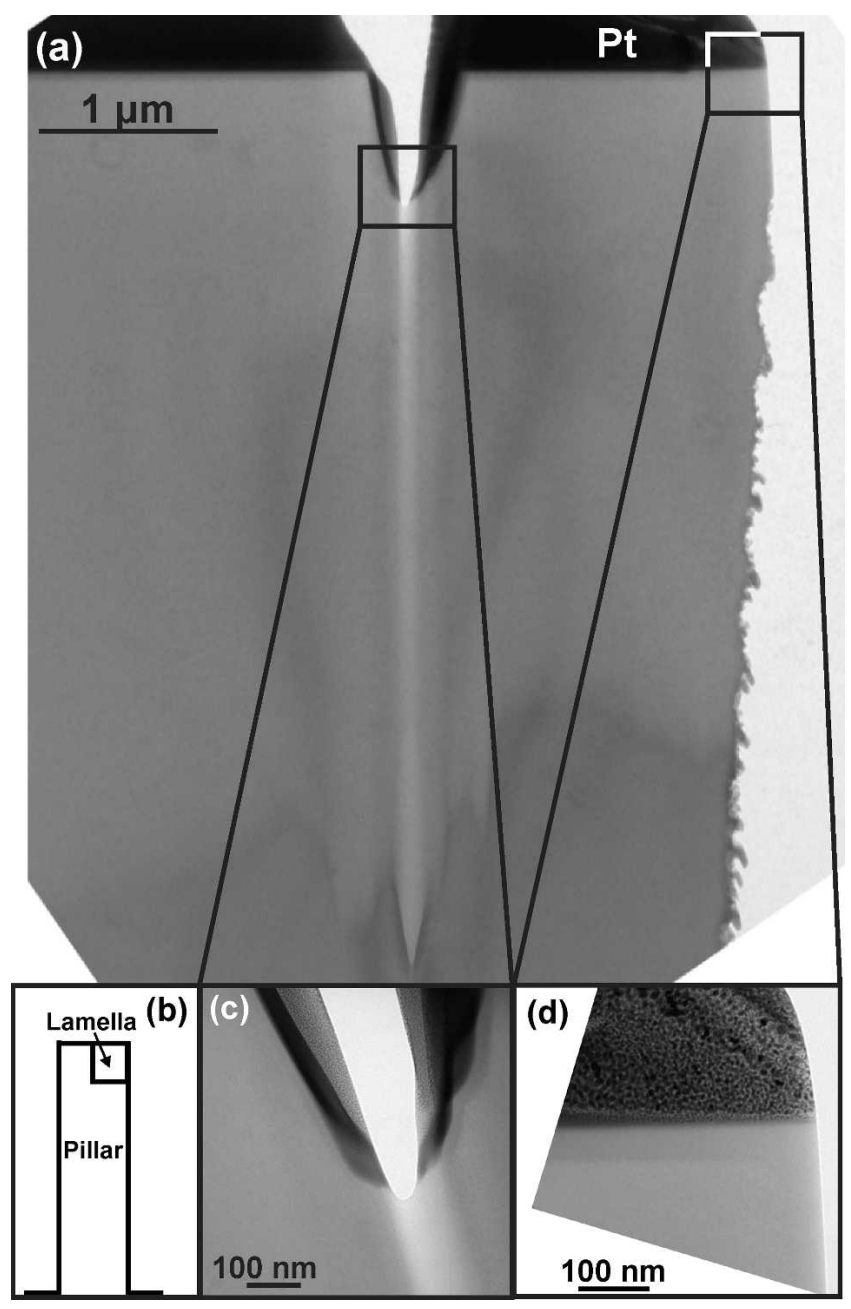

FIG. 6. TEM bright field ([110] projection) of the lamella made at the edge of a pillar subjected to a compression stress of $-4.6 \mathrm{GPa}$. (a) Overview of a dislocation-free pillar milled on the side of the pillar with a crack starting at the surface. It shows as well the platinum layer at the surface and the roughness of the side of the pillar. (b) Location of the FIB lamella at the edge of the micropillar. (c) The inset illustrates the enlargement of the crack presented and that no dislocation is seen. (d) The inset shows the edge of the pillar where no dislocation is visible.

inset (c) in Fig. 5 that additional small cracks with different orientation exist along an array of few dislocations. As the pillars were made on a (001) wafer, their axis belongs to a [001] direction and so the plane of the lamella is $\{110\}$. From these geometrical considerations, it appears that the small cracks are along $\{111\}$ planes, as expected from free surface energy. As no dislocation was observed on other parts of this lamella, it is supposed that that their nucleation was triggered by crack interaction. The diffraction pattern [Fig. 5(d)] demonstrates that no permanent phase transformation can be seen. The lamella cut at the edge of the pillar presented in Fig. 6 contains only a large crack initiated at the surface with a depth of $800 \mathrm{~nm}$, but is completely free from dislocations. Simi- larly to Fig. 5, the crack was observed previously from the SEM image taken during the FIB preparation. Figure 6(a) shows the edge of the pillar prepared according to Fig. 6(b) and the roughness coming from the preparation by the photolithography technique. No dislocation is seen, even at stress-concentrating features such as the edges of the crack, corners, or roughness on the pillar wall, and evidence of this is shown in Fig. 6(d). A small $(<50 \mathrm{~nm})$ amorphous layer on top of the pillar coming from the protective platinum layer deposition ${ }^{19,20}$ is observed in both figures. In Fig. 6(c), it is observed that the platinum layer is visible on the side of the crack but has been milled away at the bottom of the crack. This indicates that the crack was created during the experiment and that it was partly enlarged by the ion beam during the TEM sample preparation.

\section{DISCUSSION}

In situ SEM observations of microcompression tests revealed that the micropillars used in this work break mainly by brittle fracture without buckling. Only some pillars exhibited visible crack formation before rupture. ${ }^{5}$ Furthermore, earlier observations showed that pillars partly sank into the substrate during testing, which is in agreement with an elastic model of the compression behavior. $^{21}$

Compared with the results given by Moser et al., ${ }^{5}$ the engineering stress at fracture in the current experiment given in Table I is lower by $30 \%$. This discrepancy is solely due to the difference in diameter used to calculate the engineering stress. Moser et al..$^{5}$ used the pillar diameter at the location of its fracture, which is at its base $(\phi 6.6 \mu \mathrm{m})$. In this work, the diameter at the position of the laser spot was taken for stress calculation, that is, $\phi$ $7.3 \mu \mathrm{m}$ based on Figs. 1 and 3(a). Strain measurements derived from the displacement of the stack piezo are influenced by the compliance of the load cell as well as the sink-in of the pillar into the substrate,$^{21}$ which has already been addressed by Moser et al. ${ }^{5}$ These two disadvantages make the derivation of the strain in the pillars from the load-displacement data difficult. Thus, effects that depend on an accurate strain measurement are not discussed. Moreover, one should bear in mind that different volumes are probed by load-cell measurements versus Raman microscopy. With the load cell, the entire volume of the pillar contributes to the signal. In contrast, Raman microscopy measures a relatively small roughly cylindrical volume close to the surface on the side of the pillar $(\phi 2 \mu \mathrm{m})$ and a depth of $250 \mathrm{~nm}$.

The stresses calculated from the load-cell data and from the Raman method in Figs. 3(b) and 3(c) are in good agreement as they differ by less than $3 \%$. This divergence is negligible considering (i) the uncertainties in the Raman measurement (the precision of the peaks 
is on the order of $0.05 \mathrm{~cm}^{-1}$ wave numbers), (ii) the error in the load-cell measurements of the microindenter, which is around $1 \mathrm{mN}$, (iii) the error in phonon shifts from the thermal expansion due to sample heating $\left(\sim 0.025 \mathrm{~cm}^{-1} / \mathrm{K}^{18}\right)$, (iv) the error of defocusing, which is less than $\pm 0.05 \mathrm{~cm}^{-1}$ in the range of $-1 \mu \mathrm{m}<z<1 \mu \mathrm{m},{ }^{18}$ and finally (v) the accuracy in positioning the laser exactly where the diameter was measured for the stress calculation. Concerning the latter and assuming a positioning error of $1 \mu \mathrm{m}$ along the height of the pillar in Fig. 1, the cross section varies by $2.5 \%$. Figure 3(b) clearly demonstrates that the stress relaxation is reversible. This is supported by Fig. 3(c) and the TEM investigations in Figs. 5 and 6, which demonstrate that no phase transformation but only few cracks and dislocations exist within the compressed pillars to stresses up to $-4.6 \mathrm{GPa}$. Hence, it can be concluded that its behavior is mainly elastic with very little plastic deformation.

The shoulders on the low-energy side of the Raman silicon peak shown in Fig. 4 are visible in only three experiments. Two examples of such behavior are displayed in Fig. 4. It is evident from this figure that the behavior of the shoulders differs in terms of point of appearance and duration, but the band lies within 490 to $520 \mathrm{~cm}^{-1}$. Possible origins of these bands, taking into account the shift due to the compressive stress, could be assigned to either the formation of Si nanocrystals, ${ }^{22,23}$ Si-IV (hexagonal diamond structure) phases, ${ }^{10-13,24}$ or regions with extensive cracking. ${ }^{10}$ These mechanisms have been observed to result in similar peaks as shown in Fig. 4.

As the formation of nanocrystalline Si requires a large amount of plastic deformation and all specimens failed in a brittle manner, this explanation can be discarded. Moreover, phase transformation from Si-I to Si-IV can occur directly from Si-I through twin intersections and depends on the density of twins in the shear deformed material ${ }^{13,25}$; this explanation is neglected as well based on the TEM analyses in Figs. 5 and 6. Consequently, only regions with extensive cracking seem a possible explanation for the shoulder observed.

Analyzing in detail the various results presented, all pillars deformed purely elastically up to a stress of $-1.17 \mathrm{GPa}$ (prior to the appearance of a shoulder in the Raman spectrum). Real-time in situ SEM test observations and the load-displacement curves showed that no irreversible deformation or cracking occurs for pillars unloaded just before failure. However, careful postexaminations showed that in some pillars longitudinal cracking exists. ${ }^{5}$ As shown from the TEM investigation, such cracks are likely to occur at the intersection of slip bands or nucleate at surface irregularities, which is consistent with the findings of Lloyd et al. ${ }^{26}$ The crack tip is surrounded by a small zone of extremely high tensile stress, which can be determined from the stress-intensity factor $K$, according to $K=\sigma \cdot \sqrt{\pi \cdot a}$, where $\sigma$ and $a$ are the engineering stress and the crack length, respectively. The theoretical tensile strength $\left(\sigma_{\mathrm{th}}\right)$ of silicon ranges between 13 and $18.5 \mathrm{GPa}$ depending on the crystallographic direction, assuming a rule of thumb $\sigma_{\mathrm{th}}=$ $E / 10$ and making use of $E_{001}=130 \mathrm{GPa}$ and $E_{111}=$ $185 \mathrm{GPa}^{27}$ This is consistent with the literature where Hoffmann et al. ${ }^{28}$ have measured a bending failure strength of $12 \mathrm{GPa}$ for nanowires grown on a [111] silicon substrate by the vapor-liquid-solid process with diameters ranging from 100 to $200 \mathrm{~nm}$ and a length of $2 \mu \mathrm{m}$. Namazu et al. ${ }^{4}$ have also found an average bending strength of $17.5 \mathrm{GPa}$ for micromachined silicon beams along [111] direction of $6 \mu \mathrm{m}$ length and around $250 \mathrm{~nm}$ in diameter. Moreover, molecular dynamics simulations indicate even higher strengths of up to $40 \mathrm{GPa}$ are possible. ${ }^{29}$ If such a crack propagates through the laser focus spot, the Raman signal is undoubtedly affected. Actually, tensile stresses will shift the Raman peak to lower wave numbers, which is in agreement with Fig. 4. By combining the Raman with the TEM observations, one could speculate that the shoulder originates from a crack growing through the laser spot. Stress evaluation from the difference in the peak position of the shoulder and the one for the unstressed silicon and using the stress sensitivity as known from the literature, ${ }^{8,17}$ results in tensile stresses in the order of 10 to $12 \mathrm{GPa}$, which is reasonable for a highly stressed zone around a crack tip and still lower than the theoretical tensile strength. As the signal intensity of the shoulder is low, the peak position cannot be determined with high accuracy. Therefore, the calculation of the tensile stress is a rough estimation of the in-plane component of the stress tensor. As the spot size of the laser beam was $2 \mu \mathrm{m}$ with a penetration depth of about $250 \mathrm{~nm}$, the measured volume is bigger than the stress field around crack tips such as the one observed in Figs. 5 and 6. This leads to the following conclusions: (i) the intensity of the shoulder is small compared with the normal Raman peak, (ii) the lateral resolution of the microscope allows only measurement of an average tensile stress in the vicinity of the crack tip, and (iii) cracks outside the laser focus spot were not detected. Taking into account the diameter of the laser focus spot as well as the penetration depth of the laser and comparing it with the cross-sectional area of the pillar, it is found that only $2 \%$ of the crosssectional area is covered by the laser. This explains why this behavior is observed only in 5\% of the measurements. The fact that the shoulder appears only temporarily could also be explained with the laser spot size. As soon as the crack has propagated completely through the volume probed by the laser, the signal is no longer influenced by the crack since no stress exists at the crack surface. 


\section{CONCLUSIONS}

In this contribution, a novel technique to characterize the mechanical properties of silicon micropillars combining micro-Raman spectroscopy with a microindenter is presented. Tests can be performed at high data acquisition rates and also quickly after having automated the system. The main advantage of micro-Raman spectroscopy compared with other methods, including in situ SEM compression tests, is to not only gain information about the local compressive stresses, which can also be accessed during the experiment, but also to monitor locally certain microstructural details by the analysis of peak positions and shapes such as grain size, phase transformations, and stress gradients. Verifying the type of microstructural changes by TEM analysis allows determination of the point of occurrence of the microstructural changes. The method is applicable for specimen sizes from the millimeter to the submicrometer ranges. In the submicrometer range, the position accuracy of the laser with the optical microscope and the laser diameter are the only limitations.

It has been shown that the stresses derived from both the load cell and the stress-induced Raman shift agree very well with a discrepancy of less than $3 \%$. In three experiments, a Raman band appeared at 490 to $520 \mathrm{~cm}^{-1}$ for compressive stresses ranging between -1.2 and $-2.5 \mathrm{GPa}$. Based on the TEM study, it was found that the main deformation mechanism of the pillars is brittle fracture. Dislocation nucleation and movement seems to play only a secondary role and to be triggered by interaction with cracks. Phase transformations could be observed neither during (by Raman spectroscopy) nor after the deformation (by TEM). Hence, we strongly believe that the shoulders observed are caused by cracks propagating through the laser spots. This result is corroborated by comparing the theoretical stress of silicon (13-18.5 GPa) with the calculated in-plane tensile stress obtained from Raman shift (10-12 GPa).

Finally, the amalgamation of micro-Raman spectroscopy with a microindenter makes it possible to locally detect microstructural changes such as phase transformations and stress gradients induced by high compressive stress to silicon micropillars. The results demonstrate that the combination of both methods is an efficient technique not only to determine material properties in the micrometer range but also to identify the onset of crack propagation as a potential solution for process control.

\section{ACKNOWLEDGMENTS}

The authors would like to thank C. Grange for conducting the microcompression tests, L. Barbieri for micropillar preparation, and Joy Tharian for the FIB lamellae.

\section{REFERENCES}

1. F. Ericson and J.A. Schweitz: Micromechanical fracture strength of silicon. J. Appl. Phys. 68, 5840 (1990).

2. O.M. Jadaan, N.N. Nemeth, J. Bagdahn, and W.N. Sharpe: Probabilistic Weibull behavior and mechanical properties of MEMS brittle materials. J. Mater. Sci. 38, 4087 (2003).

3. S. Greek, F. Ericson, S. Johansson, and J.A. Schweitz: In situ tensile strength measurement and Weibull analysis of thick film and thin film micromachined polysilicon structures. Thin Solid Films 292, 247 (1997).

4. T. Namazu, Y. Isono, and T. Tanaka: Evaluation of size effect on mechanical properties of single crystal silicon by nanoscale bending test using AFM. J. Microelectromech. Svst. 9, 450 (2000).

5. B. Moser, K. Wasmer, L. Barbieri, and J. Michler: Strength and fracture of Si micropillars: A new scanning electron microscopybased micro-compression test. J.Mater. Res. 22, 1004 (2007).

6. E. Anastassakis, A. Pinczuk, A. Burstein, F.H. Pollak, and M. Cardona: Effect of static uniaxial stress on Raman spectrum of silicon. Solid State Commun. 8, 133 (1970).

7. E. Anastassakis, A. Canterero, and M. Cardona: Piezo-Raman measurements and anharmonic parameters in silicon and diamond. Phys. Rev. B 41, 7529 (1990).

8. I. De Wolf: Micro-Raman spectroscopy to study local mechanical stress in silicon integrated circuits. Semicond. Sci. Technol. 11. 139 (1996).

9. V.T. Srikar, A.K. Swan, M S. Ünlü, B.B. Goldberg, and S.M. Spearing: Micro-Raman measurement of bending stresses in micromachined silicon flexures. J. MEMS 12, 779 (2003).

10. A. Keiler, Y.G. Gogotsi, and K.G. Nickel: Phase transformations of silicon caused by contact loading. J. Appl. Phys. 81, 3057 $\underline{(1997)}$.

11. Y.G. Gogotsi, T. Miletich, M. Gardner, and M. Rosenberg: Microindentation device for in situ study of pressure-induced phase transformations. Rev. Sci. Instrum. 70, 4612 (1999).

12. Y.G. Gogotsi, G. Zhou, S-S. Ku, and S. Cetinkunt: Raman microspectroscopy of pressure-induced metallization in scratching of silicon. Semicond. Sci. Technol. 16, 345 (2001).

13. V. Domnich and Y.G. Gogotsi: Phase transformation in silicon under contact loading. Rev. Adv. Mater. Sci. 3, 1 (2002).

14. R. Gassilloud, C. Ballif, P. Gasser, G. Buerki, and J. Michler: Deformation mechanisms of silicon during nanoscratching. Phvs. Status Solidi A 202, 2858 (2005).

15. Z. Iqbal and S. Veprek: Raman scattering from hydrogenated microcrystalline and amorphous silicon. J.Phys. C: Solid State Phvs. 15, 377 (1982).

16. J.K. Bhardwaj and H. Ashraf: Advanced silicon etching using high density plasmas, Proceedings, Micromachining and Microfabrication Process Technology (The International Society for Optical Engineering, Austin, TX, 1995), pp. 224-233.

17. R. Rabe, J-M. Breguet, P. Schwaller, S. Stauss, F-J. Haug, J. Patscheider, and J. Michler: Observation of fracture and plastic deformation during indentation and scratching inside the scanning electron microscope. Thin Solid Films 469-470, 206 (2004).

18. K.F. Dombrowski: Micro-Raman investigation of mechanical stress in Si device structures and phonons in SiGe. Ph.D. Thesis, Brandenburgische Technische Universität Cottbus, Cottbus, Germany (2000).

19. S. Rubanov and P.R. Munroe: FIB-induce damage in silicon. J. Microsc. 214, 213 (2004).

20. L. Frey, C. Lehrer, and H. Rysell: Nanoscale effects in focused ion beam processing. Appl. Phvs. A 76, 1017 (2003).

21. H. Zhang, B.E. Schuster, Q. Wei, and K.T. Ramesh: The design of accurate micro-compression experiments. Scr. Mater. 54, 181 $\underline{(2006)}$. 
22. X.S. Zhao, Y.R. Ge, J. Schroeder, and P.D. Persans: Carrierinduced strain effect in Si and GaAs nanocrystals. Appl. Phys. Lett. 65, 2033 (1994).

23. J. Zi, H. Büscher, C. Falter, W. Ludwig, K. Zhang, and X. Xie: Raman shifts in Si nanocrystals. Appl. Phys. Lett. 69, 200 (1996).

24. G.W. Weill, J.L. Mansot, G. Sagon, C. Carlone, and J.M. Besson: Characterisation of Si III and Si IV, metastable forms of silicon at ambient pressure. Semicond. Sci. Technol. 4, 280 (1989).

25. U. Dahmen, C.J. Hetherington, P. Pirouz, and K.H. Westmacott: The formation of hexagonal silicon at twin intersections. Acta Metall. Mater. 38, 269 (1989).

26. S.J. Lloyd, J.M. Molina-Aldaregui, and W.J. Clegg: Deformation under nanoindents in $\mathrm{Si}, \mathrm{Ge}$, and GaAs examined through transmission electron microscopy. J. Mater. Res. 16, 3347 (2001).

27. M. Levinshtein, S. Rumyanstev, and M. Shur: Handbook Series on Semiconductor Parameters, Vol. 1, 1st ed. (World Scientific, Singapore, 1996), pp. 1-30.

28. S. Hoffmann, I. Utke, B. Moser, J. Michler, S. Christiansen, V. Schmidt, S. Senz, P. Werner, U. Gösele, and C. Ballif: Measurement of the bending strength of vapor-liquid-solid grown silicon nanowires. Nano Lett. 6(4), 622 (2006).

29. R. Nyilas and R. Spolenak: Orientation-dependent ductile-tobrittle transitions in nanostructured materials. Acta Mater. (2008, DOI: 10.1016/j.actamat.2008.07.051). 